\title{
Duodenal mucosal resurfacing: Multicenter experience implementing a minimally invasive endoscopic procedure for treatment of type 2 diabetes mellitus $\square$
}

(๑) $\odot \ominus$

\section{Authors}

Annieke C.G. van Baar ${ }^{1}$, Rehan Haidry², Leonardo Rodriguez Grunert ${ }^{3}$, Manoel P. Neto Galvao ${ }^{4,5}$, Raf Bisschops ${ }^{6}$, Bu Hussain Hayee $^{7}$, Guido Costamagna ${ }^{8,9}$, Jacques Deviere ${ }^{10}$, Jacques J.G.H.M. Bergman ${ }^{1}$

Institutions

1 Gastroenterology and Hepatology, Amsterdam University Medical Centers, Amsterdam, the Netherlands

2 Gastroenterology, University College Hospital, London, UK

3 CCO Clinical Center for Diabetes, Obesity and Reflux, Santiago, Chile

4 Bariatric Endoscopy service, Endovitta Institute, Sao Paulo, Brazil

5 Surgery, FMABC Medical School, Sao Paulo, Brazil

6 Gastroenterology and Hepatology, Catholic University of Leuven, Leuven, Belgium

7 Institute of Therapeutic Endoscopy, King's Health Partners, London, UK

8 Digestive Endoscopy, Fondazione Policlinico Universitario A. Gemelli - IRCCS, Rome, Italy

9 CERTT (Centre for Endoscopic Research Therapeutics and Training), Università Cattolica, Rome, Italy

10 Gastroenterology, Erasme University Hospital, Brussels, Belgium

submitted 14.7.2020

accepted after revision 30.7.2020

\section{Bibliography}

Endoscopy International Open 2020; 08: E1683-E1689

DOI 10.1055/a-1244-2283

ISSN 2364-3722

(C) 2020. The Author(s).

This is an open access article published by Thieme under the terms of the Creative Commons Attribution-NonDerivative-NonCommercial License, permitting copying and reproduction so long as the original work is given appropriate credit. Contents may not be used for commecial purposes, or adapted, remixed, transformed or built upon. (https://creativecommons.org/licenses/by-nc-nd/4.0/)

Corresponding author

Prof. Jacques J.G.H.M. Bergman, Department of Gastroenterology and Hepatology, Amsterdam University

Medical Center, Meibergdreef 9, 1105AZ Amsterdam,

The Netherlands

Fax: + 0031205669000

j.j.bergman@amsterdamumc.nl
ABSTRACT

Background and study aims Duodenal mucosal resurfacing (DMR) is an endoscopic procedure which improves insulin resistant metabolic disease, including type 2 diabetes mellitus (T2DM). The aim of this report was to evaluate the feasibility and procedural aspects of DMR and to provide more specific DMR procedural guidance for endoscopists.

Patients and methods In this international multicenter, prospective, open-label study, patients on oral anti-diabetic agents for treating T2DM underwent single DMR. DMR entails circumferential submucosal lifting followed by circumferential mucosal hydrothermal ablation using an over-theguidewire balloon catheter for lifting and ablation. For the first 28 patients a dual catheter system was used. During the study, a new integrated catheter was developed which was used for the latter 18 patients. During DMR, procedure success (complete DMR: duodenal ablation length $\geq 9 \mathrm{~cm}$ ) and procedure duration were captured.

Results Forty-six patients underwent DMR. Using the dual catheter system, a complete DMR was performed in 22 of 28 patients (79\%). In the next eighteen patients who underwent DMR with the integrated catheter, a complete DMR was performed in 15 of 18 patients (83\%). The integrated catheter facilitated the DMR procedure and resulted in a reduction in procedure time. A detailed table and video are provided for future endoscopists.

Conclusions In our multicenter study, DMR was found to be feasible in the hands of experienced endoscopists. The integrated DMR catheter was a welcome modification during the study, allowing for easier ablation administration. Further optimization of the technique would be valuable prior to widespread dissemination. 


\section{Introduction}

Worldwide, 387 million people are diagnosed with type 2 diabetes mellitus (T2DM), and the number continues to rise at an alarming rate in every country [1]. Despite lifestyle interventions and the increasing array of pharmacological therapies, most patients with T2DM fail to achieve established treatment goals to prevent complications [2]. Recently, minimally invasive endoscopic treatment options for T2DMhave emerged. Evidence from bariatric surgery and the duodenal-jejunal bypass liner highlights the importance of the small bowel, especially the duodenum, in glucose homeostasis and metabolic regulation [3]. If contact between the duodenal mucosa, bile, and nutrients is prevented or altered, then insulin sensitivity improves and $\beta$-cell function increases [4-6]. These improvements are quickly reversed when the bypassed duodenum is re-exposed to nutrients, underscoring the glucoregulatory role of the duodenum $[4,7,8]$.

Duodenal mucosal resurfacing (DMR) is a minimally invasive endoscopic procedure involving catheter-based hydrothermal ablation of duodenal mucosa [9]. DMR may offer a new minimally invasive treatment approach for insulin-resistant metabolic diseases, including T2DM, nonalcoholic fatty liver disease (NAFLD), and nonalcoholic steatohepatitis (NASH) [10-12]. The first multicenter study reported durable and substantial glycaemic improvement in patients with T2DM as well as a decrease in liver transaminase levels [10] at 24 weeks and 12 months [11]. Here we report on and evaluate the procedural feasibility of DMR. More specifically, we elaborate on DMR catheter development and improvements and endoscopic procedure optimization. In addition, we provide tips and tricks for endoscopists in order to conduct a successful DMR procedure.

\section{Patients and methods}

\section{Study design}

We evaluated endoscopic feasibility data from the first open-label, single-arm, multicenter study (NCT02413567) conducted at seven clinical sites in the EU and Chile (Academic Medical Center of Amsterdam, Netherlands; Erasme University Hospital Brussels, Belgium; Policlinico Gemelli, Catholic University of Rome, Italy; University College Hospital London, United Kingdom; CCO Clinical Center for Diabetes, Obesity and Reflux, Santiago, Chile; King's College Hospital, London, United Kingdom; and University Hospital Leuven, Leuven, Belgium) [11]. At each site, a single endoscopist performed the DMR procedure after completing initial didactic and hands-on DMR training in a porcine model. The study protocol was approved by the independent ethics committees at each center. The study was conducted in accordance with Good Clinical Practice Guidelines and the Declaration of Helsinki.

\section{Patients}

Eligible patients had T2DMand used oral glucose-lowering medication, were aged 28 to 75 years, had a body mass index between 24 and $40 \mathrm{~kg} / \mathrm{m}^{2}$ and hemoglobin A1c (HbA1c) between 59 and $86 \mathrm{mmol} / \mathrm{mol}$ (7.5\%-10.0\%). Main exclusion criteria were Type 1 diabetes (including positive GAD antibodies), use of injectable glucose-lowering medication, previous gastrointestinal surgery that could affect the ability to treat the duodenum (eg, Bilroth 2 or Roux-en-Y gastric bypass), history of pancreatitis, and upper gastrointestinal bleeding. The complete eligibility list is available in te recently published efficacy study [11]. Written informed consent was obtained from all patients at screening.

\section{Endoscopic setting}

The DMR procedure was performed under general anesthesia or deep sedation with propofol, according to local guidelines and the endoscopists' preference. First, a screening gastroduodenoscopy was conducted to ensure there were no conditions that would impede the DMR procedure (e. g., erosive esophagitis $\geq$ grade $C$, gastroduodenal ulcers, varices, strictures, or telangiectasia). Next, the papilla of Vater was marked on the contralateral duodenal wall using argon plasma coagulation (APC) or a hemostasis clip, to mark the proximal margins of the intended ablation zone. Then, a guidewire (recommended: Jagwire 0.035 " Stiff Shaft [Boston Scientific, Marlborough, Massachusetts, United States]) was placed past the ligament of Treitz to assist delivery of the DMR catheters. Fluoroscopy was used to verify the delivery and positioning of the guidewire.

\section{Initial modifications of the DMR procedure}

Prior to this multicenter study, three patients from the first-inhuman study developed a duodenal stenosis within 6 weeks after the procedure [12]. The stenoses were resolved by endoscopic balloon dilatation without further sequelae. Root cause analysis revealed that overlapping ablations and ablation of non-lifted duodenal mucosa were the likely causes for development of these duodenal stenoses after DMR. Therefore, the following modifications were made to the DMR procedure prior to initiation of the multicenter study: 1) ablation was performed from proximal to distal (instead of distal to proximal as was done in the first-in-human study) to optimize endoscopic visualization and avoid overlapping ablation zones and minimize the length of time elapsed between submucosal injection and ablation; and 2) mucosal lifting was performed more extensively to avoid ablation of non-lifted mucosa. All participating endoscopists reached a consensus on the optimal DMR procedure during a joint meeting with hands-on training in a porcine model.

\section{DMR procedure with double catheter system}

Initially, DMR was performed with two catheters (Fractyl Laboratories, Inc., Lexington, Massachusetts, United States): a submucosal expansion (SE) catheter and a hot fluid (HF) catheter. The SE catheter was tracked over the guidewire into the horizontal part of the duodenum just distal to the clip/APC mark (papilla) to measure the inner diameter of the post-papillary duodenal lumen. The endoscope (pediatric colonoscope) was positioned just proximal to the balloon at the tip of the catheter. Then three vacuum-assisted needles around the tip of the SE catheter were used to inject saline into the submucosal space. Six to nine consecutive submucosal lifting cycles were 
- Fig. 1 Integrated duodenal mucosal resurfacing balloon catheter. Single ring to be positioned just proximally of the previous ablation to perform a consequent ablation. Double ring indicates the furthest acceptable position of the endoscope.

performed to ensure submucosal lifting of the postpapillary duodenal area targeted for ablation. Between each lifting cycle, the catheter en endoscope was positioned 1 to $2 \mathrm{~cm}$ distally. Then, the SE catheter was replaced with a HF catheter (with fixed balloon diameter of 19, 21, 23 or $25 \mathrm{~mm}$ based on the SE catheter measurements). Three consecutive non-overlapping hydrothermal ablations of the lifted area were performed with a $3-\mathrm{cm}$ HF balloon under endoscopic visualization. Between each ablation cycle, the catheter and endoscope were positioned $3 \mathrm{~cm}$ distally. A complete DMR procedure was defined as three ablations corresponding with a 9-cm circumferential ablation of post-papillary duodenal mucosa.

\section{DMR procedure with integrated catheter}

During the course of this study, a single catheter ( $>$ Fig. 1 ) became available that integrated submucosal lift and hydrothermal ablation functions. The novel catheter eliminated the need for catheter exchanges during the procedure and helped ensure that ablation was performed immediately after submucosal lift of the same segment of duodenum ( $\triangleright$ Video 1 ). The DMR catheter was introduced over the guidewire and positioned just distally of the marking of the papilla in the duodenum. The tip of the endoscope (pediatric colonoscope) was positioned just proximal to the balloon guided by the reference markers on the catheter. The single integrated catheter has three vacuumassisted submucosal injectors around a 2-cm ablation balloon at its distal end to enable lifting of the duodenal mucosa. To ensure optimal lifting prior to mucosal ablation, every second lifting cycle was followed by a circumferential mucosal ablation. The catheter was advanced proximally $1 \mathrm{~cm}$ distally after each lifting or lifting-ablation cycle. Five ablations were performed to achieve a total duodenal ablation length of $10 \mathrm{~cm}$, defining a complete DMR procedure.

\section{Post-procedural care}

Patients were discharged within 24 hours after the procedure, depending on the local center's guidelines. Prior to discharge, patients were prescribed a 2-week post-procedure diet that progressed from clear liquids to solid foods to facilitate mucosal healing. The follow-up protocol and efficacy outcomes can be appreciated from our recently published study [11].

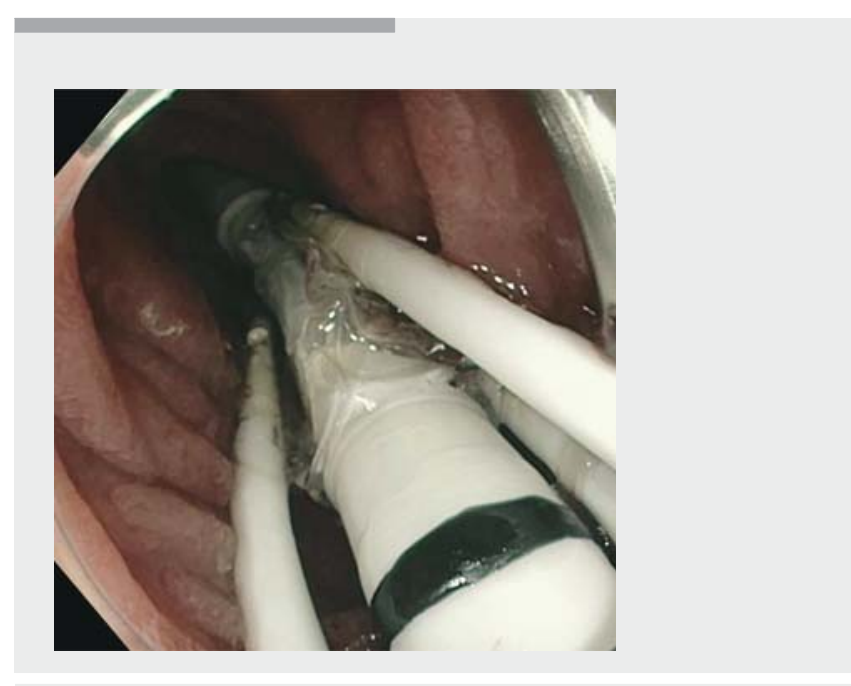

$\checkmark$ Video 1 Stepwise explanation of endoscopic duodenal mucosal resurfacing procedure.

\section{Outcomes}

Feasibility endpoints included successful completion of submucosal expansion and mucosal ablation, defined as three (double catheter system with ablation balloon of $3 \mathrm{~cm}$ ) or five (single catheter system with ablation balloon of $2 \mathrm{~cm}$ ) ablations and procedure time (calculated from the time of insertion of the endoscope until removal of the guidewire and catheter). Data are presented as mean (standard deviation [SD]) or percentage where applicable. We created a table with tips and tricks for future endoscopists to facilitate an optimal procedure.

\section{Results}

\section{Procedure time}

In total, 46 patients underwent DMR. The first 28 patients who received double-catheter DMR had a mean $(S D)$ procedure time of 96 (34) minutes. Once available, the single catheter was used for all subsequent 18 patients, which reduced the mean DMR procedure time significantly to $71(23)$ minutes $(P=.031)$.

\section{Successful completion}

The DMR procedure was completed (i.e., 3 ablation zones totaling $9 \mathrm{~cm}$ ) in 22 of 28 patients (79\%) in the double-catheter group. The full DMR treatment was not performed in six patients, due to catheter failure $(n=4)$, tortuous anatomy $(n=1)$, and difficulty with catheter positioning $(n=1)$. In the single integrated catheter group, the DMR procedure was completed (i. e., 5 ablation zones totaling $10 \mathrm{~cm}$ ) in 15 of 18 patients ( $83 \%$ ). Full DMR treatment was not possible in three patients due to difficulty with catheter handling and positioning.

A stepwise explanation of the DMR procedure, including challenges and advice for how to overcome them, is provided in $>$ Table 1. 
- Table 1 Tips and tricks for a successful DMR procedure.

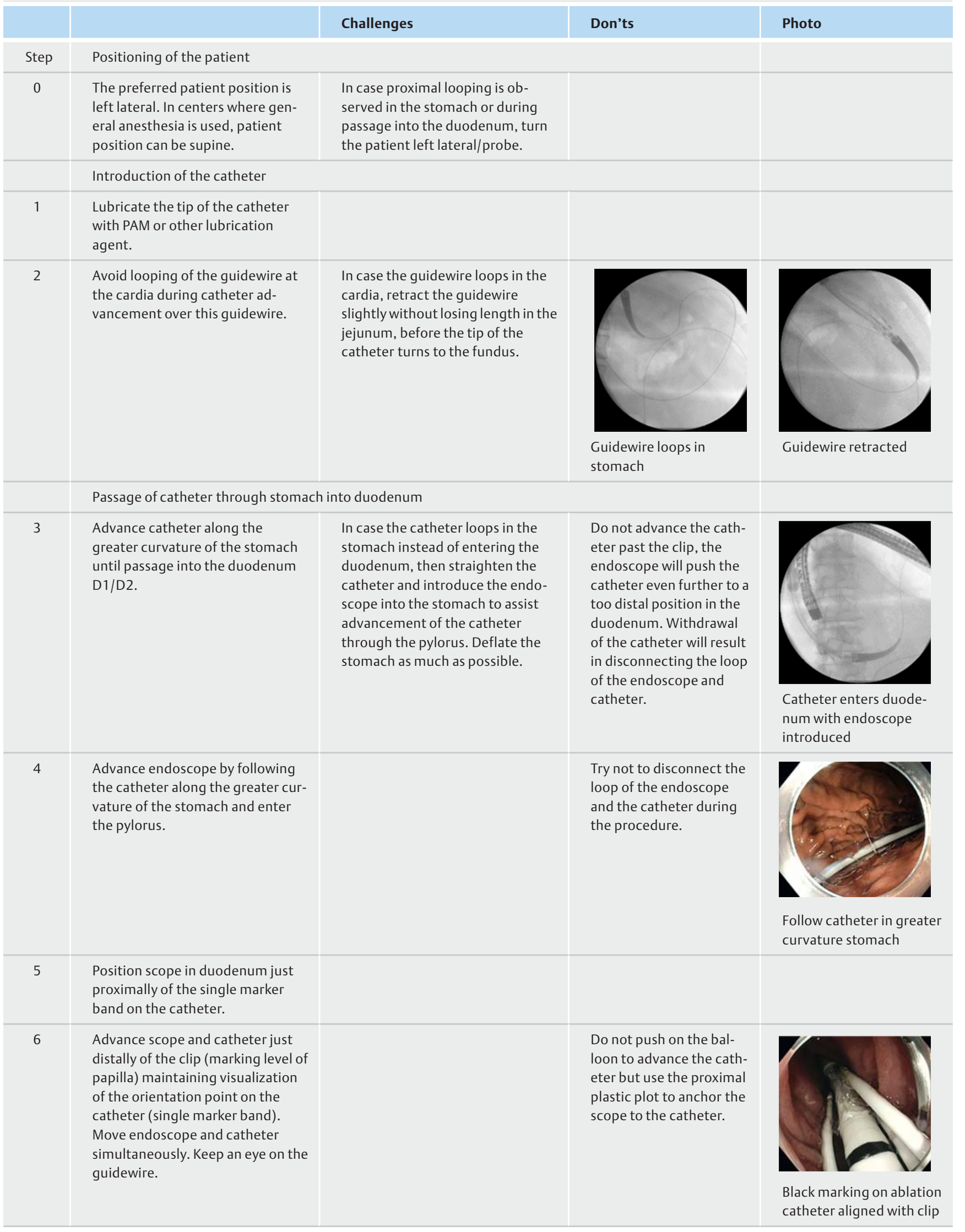


Table 1 (Continuation)

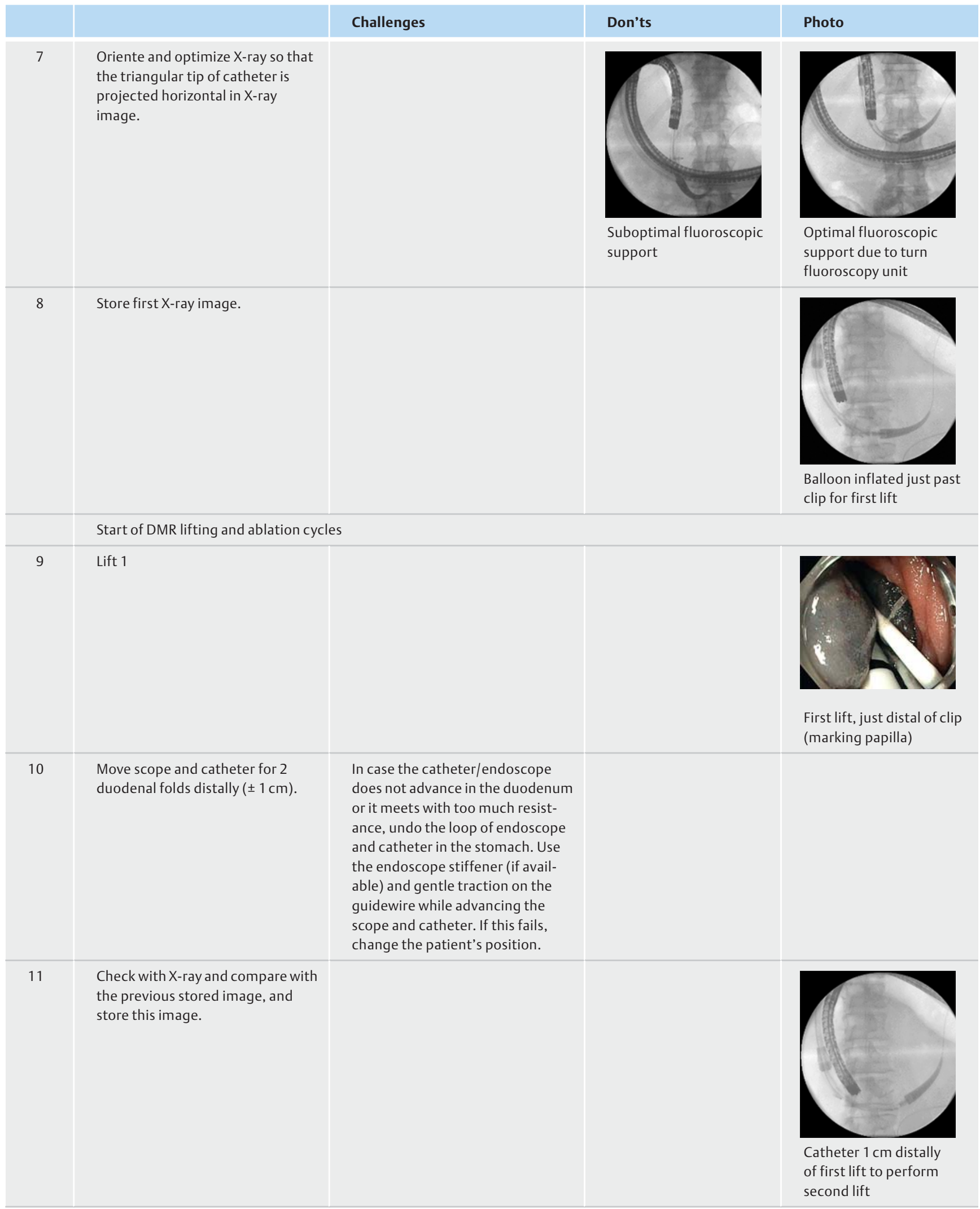


$>$ Table 1 (Continuation)

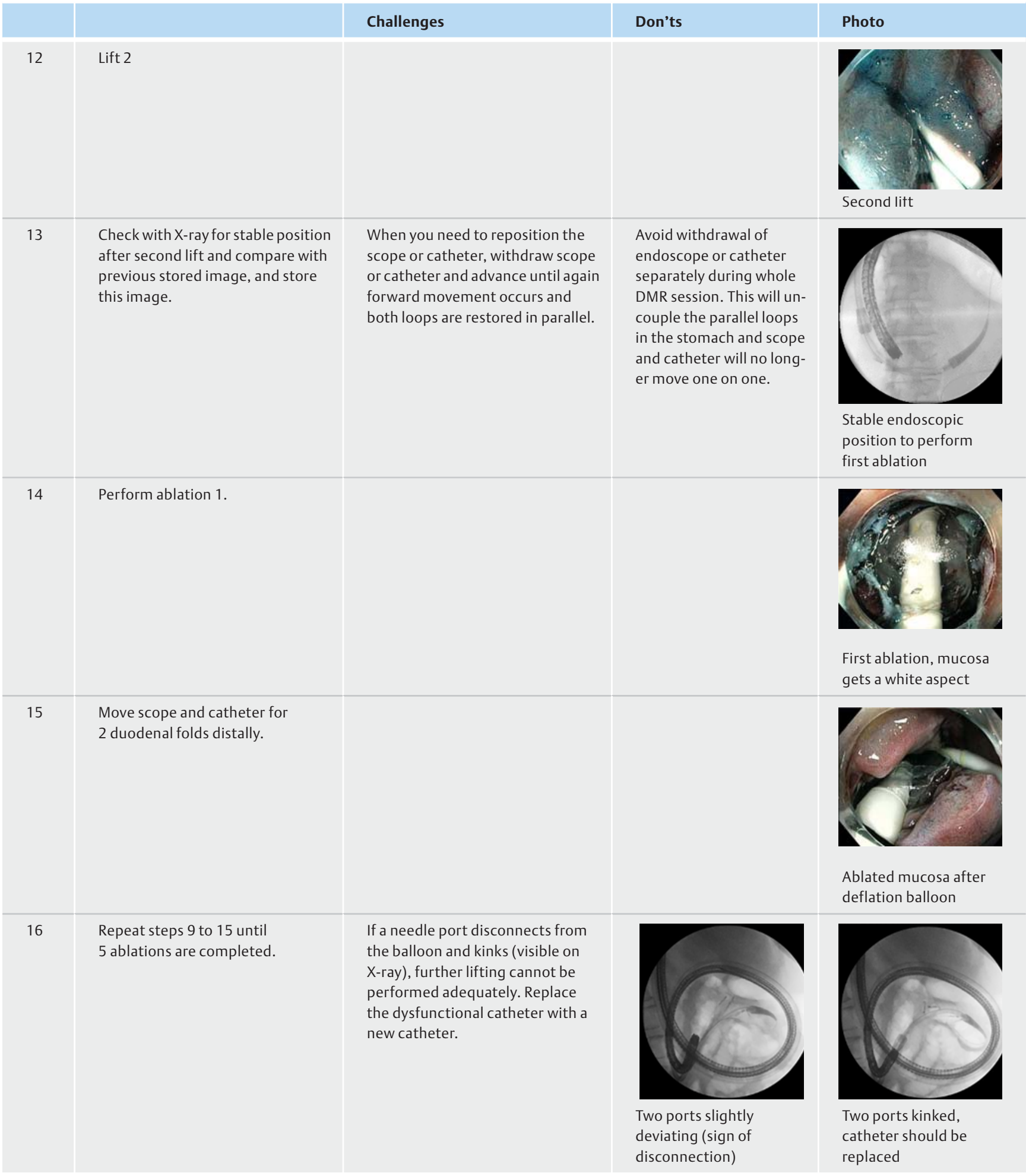

DMR, duodenal mucosal resurfacing. 


\section{Discussion}

DMR is a promising minimally invasive endoscopic procedure that appears to elicit robust and sustained improvements in metabolic parameters after a single treatment in patients with T2DM. Data from the first international, multicenter study suggest that DMR is feasible, safe, and effective in patients with T2DM that is suboptimally controlled with oral glucose-lowering medication with a considerable glucose lowering effect and a reduction in hepatic transaminase levels $[10,11]$. Reported AEs are reassuringly mild and consistent with what is observed with other general upper gastrointestinal therapeutic endoscopic approaches. No cases of duodenal stenosis were reported after the initial DMR procedure modifications prior to this study.

\section{Conclusions}

The goal of the multicenter study was to deliver a uniform DMR to the T2DM study population. During the study, the introduction of a new, single DMR catheter resulted in an overall reduction in procedure time and increased ability to perform a complete DMR procedure. DMR appears to be a safe endoscopic procedure which is feasible in the hands of experienced endoscopists. With the introduction of the integrated DMR catheter during this study, the system matured and is ready for more extensive studies and possibly also for the first clinical applications. However, a procedure success rate of $83 \%$ indicates that technical feasibility is not a given and a reduction in procedure time is desirable. Further optimization of the technique would be valuable prior to widespread dissemination. Our video and the table of practical advice from experienced DMR endoscopists are beneficial tools to increase success rate and reduce procedure time, especially for newly trained DMR endoscopists.

Further clinical investigations, including liver imaging and histology, are currently underway and a double-blind, shamcontrolled, randomized trial is being finalized. Mechanistic interrogation of the DMR effect also is underway in both animals and human subjects.

\section{Competing interests}

Dr. Rodriguez-Grunert has received honorarium for consultancy from Fractyl Laboratories. Dr. Galvao Neto reports honorarium from Fractyl Laboratories (proctor), GI Dynamics (proctor, scientific advisory board member), GI Windows (consultant), Apollo EndoSurgery (faculty in training courses, consultant, proctor), USGI (consultant, proctor), Colubris Mx (consultant, proctor), Keyron (scientific advisory board member), Ethicon EndoSurgery (faculty in training courses, consultant), Meditronics (faculty in training courses, consultant), Olympus (faculty in training courses, consultant). Dr. Hayee has received funding/grant support from Olympus, Fujifilm, Fractyl Laboratories and honorarium for consultancy from Takeda Pharmaceuticals UK, Fujifilm, Atlantic Pharmaceuticals, Endotools. Dr. Deviere has received funding/grant support from Fractyl Laboratories Inc. for IRB approved studies. Dr. Bergman received research support from Fractyl Laboratories Inc. for IRB approved studies and received a consultancy fee for a single advisory board meeting of Fractyl in September 2019. This study was funded by Fractyl Laboratories.

\section{References}

[1] da Rocha Fernandes J, Ogurtsova K, Linnenkamp U et al. IDF Diabetes Atlas estimates of 2014 global health expenditures on diabetes. Diabetes Res Clin Practice 2016; 117: 48-54

[2] Wong K, Glovaci D, Malik S et al. Comparison of demographic factors and cardiovascular risk factor control among U.S. adults with type 2 diabetes by insulin treatment classification. J Diabetes Complications 2012; 26: 169-174

[3] van Baar ACG, Nieuwdorp M, Holleman F et al. The duodenum harbors a broad untapped therapeutic potential. Gastroenterology 2018: doi:10.1053/j.gastro.2018.02.010

[4] Rubino F, Forgione A, Cummings DE et al. The mechanism of diabetes control after gastrointestinal bypass surgery reveals a role of the proximal small intestine in the pathophysiology of type 2 diabetes. Ann Surgery 2006; 244: 741-749

[5] Pories W], Albrecht RJ. Etiology of type II diabetes mellitus: role of the foregut. World J Surgery 2001; 25: 527-531

[6] Salinari S, Carr RD, Guidone C et al. Nutrient infusion bypassing duodenum-jejunum improves insulin sensitivity in glucose-tolerant and diabetic obese subjects. Am J Physiol Endocrinol Metabol 2013; 305 : E59-E66

[7] Dirksen C, Hansen DL, Madsbad S et al. Postprandial diabetic glucose tolerance is normalized by gastric bypass feeding as opposed to gastric feeding and is associated with exaggerated GLP-1 secretion: a case report. Diabetes Care 2010; 33: 375-377

[8] Betzel B, Koehestanie P, Homan J et al. Changes in glycemic control and body weight after explantation of the duodenal-jejunal bypass liner. Gastrointest Endosc 2017; 85: 409-415

[9] Haidry RJ, van Baar AC, Galvao NetoMP et al. Duodenal mucosal resurfacing: proof-of-concept, procedural development, and initial implementation in the clinical setting. Gastrointest Endosc 2019: doi:10.1016/j.gie.2019.03.024

[10] van Baar ACG, Beuers U, Wong K et al. Endoscopic duodenal mucosal resurfacing improves glycaemic and hepatic indices in type 2 diabetes: 6-month multicentre results. JHEP Rep 2019; 1: 429-437

[11] van Baar ACG, Holleman F, Crenier L et al. Endoscopic duodenal mucosal resurfacing for the treatment of type 2 diabetes mellitus: one year results from the first international, open-label, prospective, multicentre study. Gut 2020; 69: 295-303

[12] Rajagopalan H, Cherrington AD, Thompson CC et al. Endoscopic duodenal mucosal resurfacing for the treatment of type 2 diabetes: 6-month interim analysis from the first-in-human proof-of-concept study. Diabetes Care 2016; 39: 2254-2261 